\title{
Learning to read aligns visual analytical skills with grapheme-phoneme mapping: evidence from illiterates
}

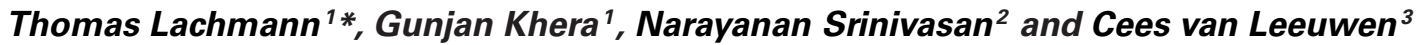 \\ Center for Cognitive Science, Cognitive and Developmental Psychology Unit, University of Kaiserslautern, Kaiserslautern, Germany \\ ${ }^{2}$ Centre of Behavioural and Cognitive Sciences, University of Allahabad, Allahabad, India \\ ${ }^{3}$ KU Leuven, Experimental Psychology Research Unit, Laboratory for Perceptual Dynamics, Leuven, Belgium
}

\section{Edited by:}

Michael Petrides, Montreal

Neurological Institute and Hospital,

Canada

\section{Reviewed by:}

Paul M. Nealen, Indiana University of PA, USA

Rosana M. Tristão, University of London, UK

\section{*Correspondence:}

Thomas Lachmann, Center for Cognitive Science, Cognitive and Developmental Psychology Unit, University of Kaiserslautern, E.-Schroedinger-Str. 57, 67663 Kaiserslautern, Germany. e-mail: lachmann@rhrk.uni-kl.de
Learning to read puts evolutionary established speech and visual object recognition functions to novel use. As we previously showed, this leads to particular rearrangements and differentiations in these functions, for instance the habitual preference for holistic perceptual organization in visual object recognition and its suppression in perceiving letters. We performed the experiment in which the differentiation between holistic non-letter processing and analytic letter processing in literates was originally shown (van Leeuwen and Lachmann, 2004) with illiterate adults. The original differentiation is absent in illiterates; they uniformly showed analytic perception for both letters and non-letters. The result implies that analytic visual perception is not a secondary development resulting from learning to read but, rather, a primary mode of perceptual organization on a par with holistic perception.

Keywords: letter recognition, object recognition, literacy, reading acquisition, dyslexia, flanker, illiterate participants, grapheme-phoneme conversion
Reading is a secondary process; learning to read depends on functional coordination of two established skills: visual object perception and the ability to use spoken language. Whereas, the former emerges at a much earlier stage than the second, both are well entrenched in human evolution. In learning to read, these skills are (1) recruited, (2) modified, (3) coordinated, and finally the coordinated process is (4) automated, in order to enable skillful, fluent reading (Lachmann, 2002). As a result, letters are detected and processed automatically in a cross-modal fashion (Blomert, 2011). To establish such a cross-modal representation requires long training, possibly 3-4 years of practice. Suboptimal automatization of this functional coordination may lead to reading disability (Lachmann et al., 2009; Blomert, 2011).

When successful, as a result of reading practice a differentiation in perceptual processing emerges (van Leeuwen and Lachmann, 2004; Burgund et al., 2006, 2009; Pegado et al., 2011). Whereas children whose reading skills are not yet fully automated process letters and similar geometric shapes in the same, holistic fashion: surrounding irrelevant visual information is uniformly assimilated with target letters and shapes in the early stages of visual feature binding, adult skilled readers, while still processing the shapes holistically, treat letters analytically: surrounding irrelevant visual context is ignored (Lachmann and van Leeuwen, 2004, 2008a) or even actively suppressed (van Leeuwen and Lachmann, 2004).

As readers acquire the differentiation in letter versus non-letter processing, the question could be asked: what perceptual skills do they lose (Serniclaes et al., 2005; Dehaene and Cohen, 2007; Lachmann and van Leeuwen, 2007; Blomert, 2011)? There is evidence that might be taken to suggest that normal reading involves losing the ability to process letters holistically. With letters and dot-patterns in a non-lexical same-different task, symmetry in dot patterns benefitted both normal reading children and their dyslexic peers, whereas symmetry in letters benefitted the dyslexics but not the normal readers. As a result, normally reading children were slower for letters in this task than dyslexics (Lachmann and van Leeuwen, 2007). On the other hand, skills seemingly lost while in transition to fluent reading might resurface later when reading is fully integrated into our system. The contrasting strategies of analytic processing for letters versus holistic processing for shapes in a classification task gave way to uniformly holistic processing, once the task required that (van Leeuwen and Lachmann, 2004). Thus, literate adults cannot be said to have lost the ability to process letters holistically (see also Schwarzer et al., 2010). Rather, they seem to refrain from it habitually.

Perhaps we are not losing perceptual skills while learning to read, but are acquiring habits that sometimes lead to suboptimal performance on specific tasks (as in Lachmann and van Leeuwen, 2007). We may consider whether the specific perceptual strategies we gain from learning to read are likewise habitual preferences rather than newly acquired perceptual skills. Our previous research has remained inconclusive about whether prior to achieving letter-specific analytic processing, children are incapable of analytic processing, and hence this skill emerges from learning to read or, alternatively, whether this skill is already present, and is recruited for processing letters. On the one hand, children early in this process show uniformly holistic processing for letters and non-letters (Lachmann and van Leeuwen, 2008a). But on the other hand, certain subgroups of dyslexics in this study showed evidence of processing letters analytically, albeit with 
extreme effort. This might well be a particular consequence of the training these children have been receiving in school. However, from these results it cannot be determined whether children have learned analytic visual processing from learning to read.

To properly investigate the question what is lost or gained from learning to read (Dehaene and Cohen, 2007), we studied a group of adults who never learned to read. We compared these to a group of adult skilled readers, using the classification task in which the differentiation in processing between letters and nonletters was originally found (van Leeuwen and Lachmann, 2004). In this task, target letters and non-letters were shown either isolated or surrounded by a task-irrelevant shape (see Figure 1, for an example). Non-letters were classified faster if the target and its surrounding were form-congruent as compared to when they differed in shape, i.e., when both were form-incongruent. This is an example of the well-known congruence effect (Pomerantz and Pristach, 1989; Bavelier et al., 2000); and can be related to early and mid-level visual perception (van Leeuwen and Bakker, 1995; Boenke et al., 2009). The congruence effect indicates, in an operationally specific sense, holistic perceptual grouping: the surrounding visual information is bound to the target, and is processed faster if both call for the same response. For letter targets, however, the opposite result was found: letters were categorized faster when surrounded by an incongruent non-target than when the non-target was congruent, i.e., a negative congruence effect.

The observed dissociation was considered a product of a specific analytic strategy optimized during learning to read in order to guarantee a rapid grapheme-phoneme mapping. Binding irrelevant visual information from the surrounding would disturb this fast mapping. The visual structure of the surrounding shape is therefore suppressed (Lachmann, 2002). Doing so is more difficult for congruent than for incongruent items, resulting in negative congruence effects (Briand, 1994; van Leeuwen and Bakker, 1995; Bavelier et al., 2000). Thus, we may conclude that in the early or intermediate stages of visual perception, skilled readers process letters using a unique analytic encoding strategy whereas for processing shapes a holistic processing strategy is applied.

For adults who have never learned to read and are unfamiliar with the alphabet, we should not expect such dissociation. Since they are not able to differentiate letters from non-letters, they will process both letters and non-letter shapes with one and the same strategy (e.g., Dehaene et al., 2010b). The question is: will that be a holistic or an analytic strategy? If the former, we may conclude that the analytic strategy is a secondary processing strategy; if it is an analytic strategy we should conclude that both analytic and holistic processing are intrinsic, primary strategies of the visual system.

\section{METHOD \\ PARTICIPANTS}

Fifty-eight adults participated in this experiment. All of them reported normal vision and hearing. The participants were informed about the reason for this investigation, that participation is voluntary and that they are free to withdraw their participation at any time while keeping their reward. The ethical committees of both the University of Allahabad and the University of Kaiserslautern gave their approval to the experiment.

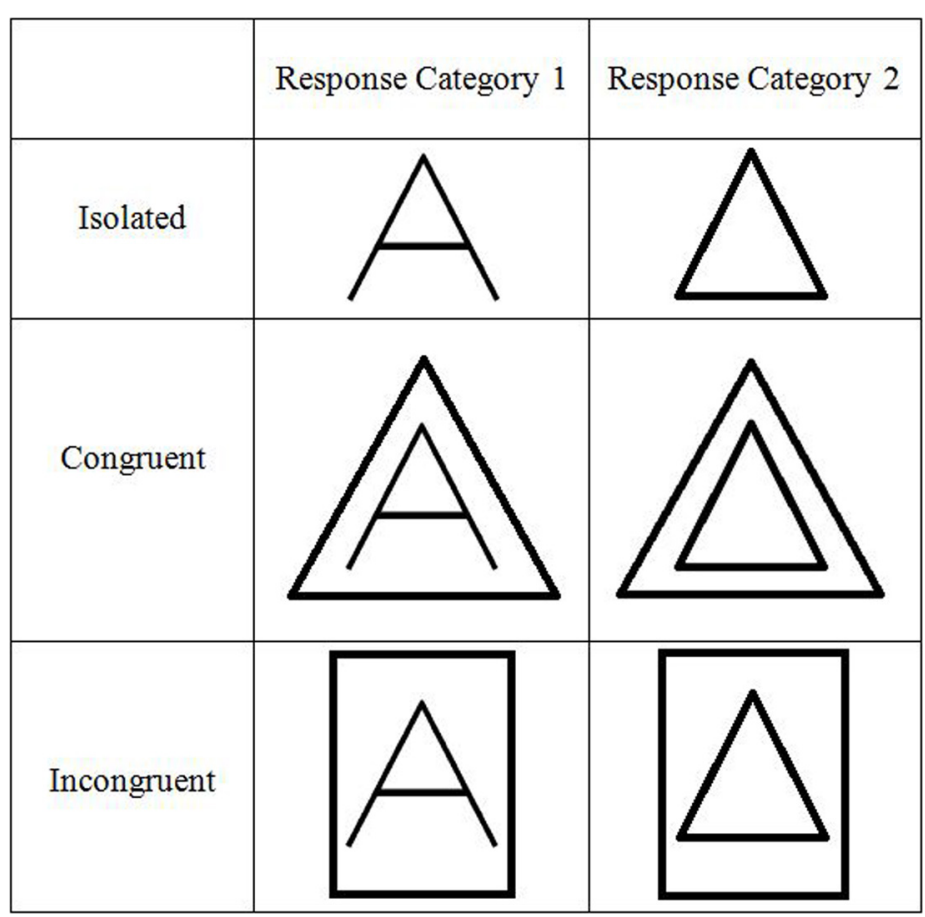

FIGURE 1 | Example for a letter target (left column) and a similar shape target (right column) as presented isolated (first line), surrounded by a form-congruent (second line) or a form-incongruent non-target in van Leeuwen and Lachmann (2004). 
The control group consisted of 26 Indian students (seven females), aged between 22 and 29 years $(M=26$ years, $\mathrm{SD}=1.97)$ from the University of Kaiserslautern, Germany. All of them were able to read fluently and to write in English. They gave their consent to the participation in written form and received 5 Euros (equivalent of 6.5 USD) for performing in this experiment.

The experimental group consisted of 32 Indian illiterates (18 females), aged between 20 and 31 years $(M=28$ years, $\mathrm{SD}=3.71$ ), from a suburb of Jasra village (Gadaiya Kalan, approximately 450 inhabitants, agriculture area), located about 35 $\mathrm{km}$ south of the city of Allahabad (Uttar Pradesh). These participants have been living in the village since they were born. Because there was no public school close by when they were children, none of them attended schooling. All participants are involved in farming and sell their products to external dealers coming to the village. There is no library in the village, nor is there any access to newspapers. Participants of this group received 150 Rs (equivalent to about 3 USD) for completing the experiment. This amount is about equivalent to an average daily wage and covers their loss in income for the day, when participating in the experiment. All these participants reported that they are not able to read neither English nor Hindi, do not speak English, and are not familiar with Latin alphabets. These participants gave their consent to the participation in spoken form.

Prior to the experiment illiterate participants' familiarity with the alphabet was determined by using a simple paper and pencil letter identification task, which included 72 letters and non-letter items, aligned in six rows. Each row contained four letters and eight non-letters, so in total 24 different letters and 48 different non-letters were displayed. Non-letters were constructed in a way that they consisted of the same segments as, but do not look too similar to Latin nor to Hindi letters (see Figure 2). Note, that nonletters used in this pre-task were not the geometrical shapes used in the experiment. Participants were instructed to mark those four items in each row of which they think they were letters. They were informed that payment does not depend on this performance. In case they were unable to decide (typical statement: "this all looks English to me"), they were instructed to guess.

The mean number of correct responses in the questionnaire was $10.35(\mathrm{SD}=4.4)$. Guessing rate was calculated on the basis of the hyper-geometric distribution $[X \sim \operatorname{Hyp}(n, H, F)]$; with $n$ is the number of guesses, $H$ is the number of possible hits, and $F$ the number of possible false alarms. Accordingly, the expected value of the guess rate is $E(X)=n \times[H /(H+F)]=1.33$ correct responses per row. For six rows the total $E(X)$ is $6 \times E(X)=8$. Taking $E(X) \pm 1 \mathrm{SD}(X)$ as criterion, with $\mathrm{SD}(X)=4.82$, we accepted all participants of this group who identified between 0.77 and 15.23 letters correctly. Alternatively, guessing rate may be calculated on the basis of Tschebyscheff-inequality and the
Gaussian, with the criterion $p<0.5 \%$, which would have resulted in a range of $0-17.44$ hits. However, we adopted the first criterion as being more conservative. On this basis, one participant, who had identified zero letters correctly, was excluded from further analyses. Another participant did not finish the test and left the setting. The rest of the participants constitute the sample described above.

\section{MATERIAL}

As in van Leeuwen and Lachmann (2004, Experiment 4), there were 24 unique stimuli. They consisted of targets: the four capital letters $A, H, L, C$; and the four geometrical shapes square, triangle, rectangle, circle, each of which was shown either in isolation or surrounded by a congruent or incongruent non-target geometrical shape which were slightly enlarged versions of the triangle, square, rectangle, and circle targets. For instance, $A$ in isolation, $A$ surrounded by a triangle (congruent condition), $A$ surrounded by a rectangle (incongruent condition).

The stimuli were scaled to an imaginary $50 \times 50 \mathrm{~mm}$ matrix, and the surrounding shapes to an imaginary $80 \times 80 \mathrm{~mm}$ matrix. Stimuli were presented in black $\left(0.29 \mathrm{~cd} / \mathrm{m}^{2}\right)$ on a Laptop screen set to white $\left(27.3 \mathrm{~cd} / \mathrm{m}^{2}\right)$ at about $50 \mathrm{~cm}$ distance, resulting in a visual angle of $3^{\circ}$ without, and $4.5^{\circ}$ with surrounding.

\section{PROCEDURE}

Literate participants performed in a laboratory room at the university. For the illiterate participants we rented a room in a private house in the village. The room was prepared in such a way that conditions were as similar to the laboratory as circumstances allowed. Electricity was guaranteed by using a mobile generator. The same portable computer was used for both groups. Participants of both groups were seated comfortably in the dimly lit environment. There was no head fixation.

The illiterate participants reported to have never used a computer before. Therefore, before instruction, they were familiarized with the computer and with the choice reaction task. They were presented with a set of short practice sessions, in which green and red dots were presented at the screen. In the first session participants were instructed to press a response key whenever a dot appears at the screen (simple reaction). In the second session a key press was required only if a red dot appeared (go/no-gotask) and, finally, in the third session the choice reaction task was trained: for red dots one key was to be pressed and one for green dots another. Prior to the experiment, literate and illiterate participants performed 12 warm up trials with the same task as used in the experiment. During practice and warming up sessions, feedback was given by presenting a yellow smiley after correct responses and a black cross after wrong responses.

Six different subsets of stimuli were presented to participants in a counterbalanced fashion (as in the original experiment,

\section{XMX $X \mathrm{H} \triangle F \& 55 T$}

FIGURE 2 | The fourth out of six rows used in the paper and pencil letter identification test. 
see Figure 2). For each individual, the stimuli were restricted to two letters and two shapes. Letters and shapes were pairwise similar, e.g., an $A$ and a triangle. They were assigned in a counterbalanced manner to two different response categories: for instance, Category 1 was an " $A$ or a Rectangle" versus Category 2 was a " $L$ or a Triangle" (Selection 1 in Figure 2). Only one stimulus was shown at a time, either with or without surrounding. The task was to decide, as fast as and accurate as possible, whether the shown target belongs to Response category 1 or 2 , ignoring the surrounding if it appears. No feedback was given. Note that letters and shapes that are similar to each other in shape, such as the $A$ and the triangle, were always assigned to different response categories (see example in Figure 3, Selection 1-3). Thus, in order to solve the task, phonological coding of the letters would be useful to distinguish between response categories, but is not necessary. In skilled readers this design was found to implicitly trigger a distinction between letters and shapes (van Leeuwen and Lachmann, 2004).

A speeded choice reaction task was required according to the response categories displayed in Figure 3, by pressing either the left or the right button marked on the keyboard of a portable computer. The four stimuli were shown to the participant prior to the experiment along with the assignment of stimuli to response buttons, e.g., if A or square press left button and if L or triangle, push right button. It was emphasized that the surrounding, if it occurs, is not relevant for the task and should be ignored.

Altogether each individual performed on 720 trials: 4 targets $\times 3$ conditions (isolated, congruent surrounding, incongruent surrounding), with 60 repeated measures. Trials were randomized, having 12 breaks in between. For illiterates the experiment took about one hour including the letter test, instruction and practice; for controls it took about half an hour.

\section{RESULTS}

Reaction times (RT) for correct responses and error rates were analyzed after rejecting outliers, which were $0.5 \%$ for the criterion RT $<145 \mathrm{~ms}$, and $0.3 \%$ for the individual criterion RT $>\mu_{n}+$ $6 \sigma_{n}$. ( $\mu_{n}=$ individual mean RT; $\sigma_{n}=$ individual mean SD).

For illiterates mean error rate was $3.3 \%$ and ranged from 0.4 to $15 \%$, three participants showed an error rate higher than $10 \%$. Mean RT was $980 \mathrm{~ms}(\mathrm{SD}=455 \mathrm{~ms})$.

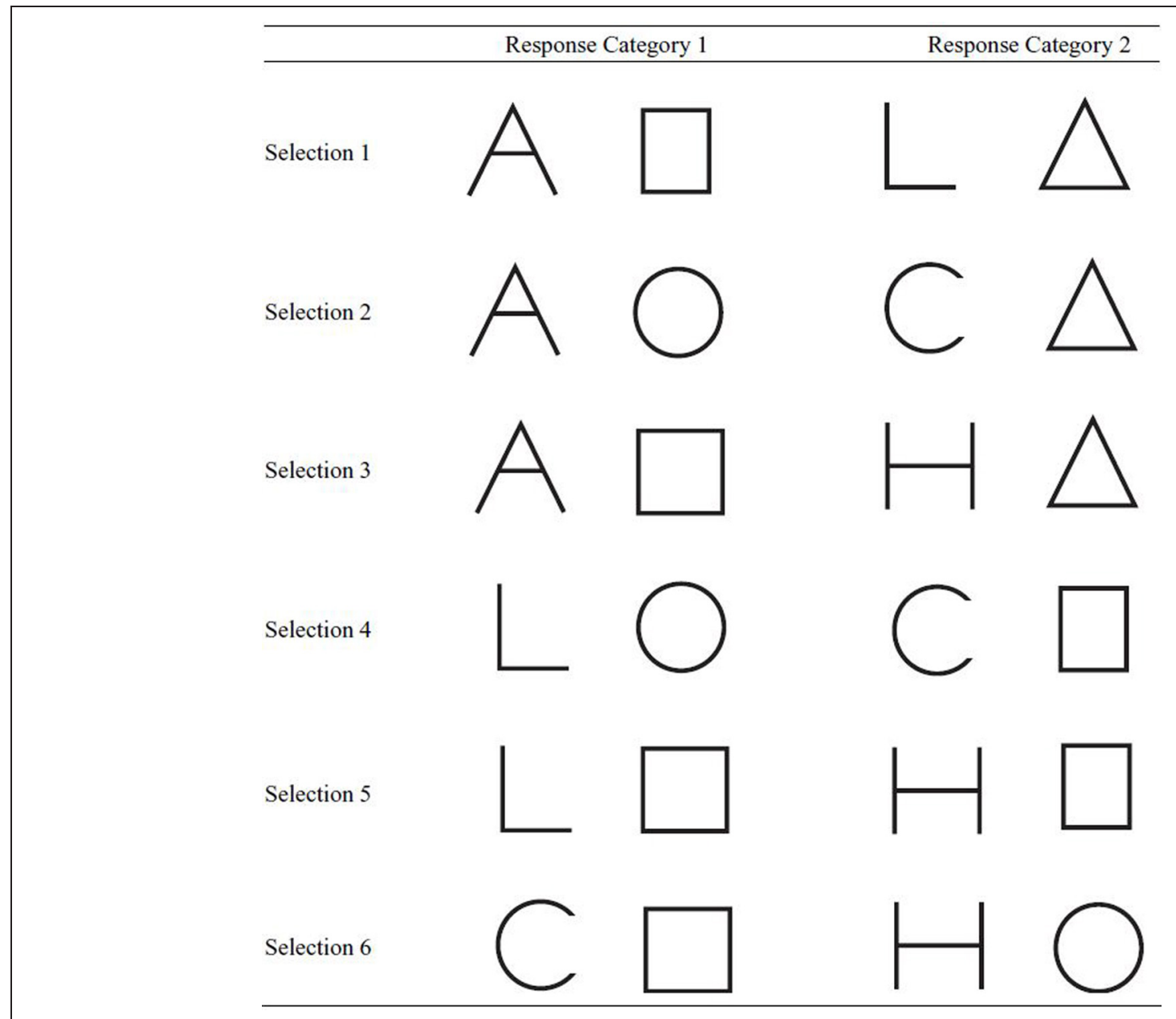

FIGURE 3 | Six selections of stimuli used in the experiment for individual participants. Further explanations in the text. 
For literates, mean error rate was 3.1\%, significantly below the $7.3 \%$ in the original study with German adults, $F_{(1,49)}=10.1$, $p<0.01$, and ranged from 0.1 to $10 \%$. The error rate did not differ from that of illiterate participants of the present study. Mean RT was $454 \mathrm{~ms}(\mathrm{SD}=186)$, which did not differ from German adults in the original study ( $477 \mathrm{~ms}, \mathrm{SD}=126)$ but is significantly lower, $F_{(1,56)}=111.2, p<0.001$, than that in illiterates, whose RTs are more than double. Only two literate participants had average RTs over $600 \mathrm{~ms}$ and only one had a higher mean RT than the fastest participant of the illiterate group.

Because there was no speed-accuracy trade-off in evidence, i.e., individual mean error rates and individual mean RTs were not correlated $(r<0.01)$, we will report Analyses of Variance (ANOVA) for RT only, using GreenHouse-Geisser correction for $p$-levels; uncorrected degrees of freedom will be reported. Since mean RTs of illiterates were nearly entirely outside the range of those of normal adult readers (Lachmann and van Leeuwen, 2004, 2008b; van Leeuwen and Lachmann, 2004; Jincho et al., 2008), ANOVAs were run for each group separately.

For the illiterates group, a two-factors repeated measures ANOVA with Congruence (isolated, congruent surrounding, incongruent surrounding) and Material (letter vs. shape) as within-participant factors revealed a main effect for Congruence, $F_{(2,62)}=5.86, p<0.01$, but not for Material $(F<1)$. No interaction was in evidence $(F<1)$. Congruent items $(995 \mathrm{~ms}$, $\mathrm{SD}=475)$ were slower than isolated ones $(967 \mathrm{~ms}, \mathrm{SD}=458)$, $F_{(1,31)}=5.67, p<0.05$, and slower than incongruent ones $(976 \mathrm{~ms}, \mathrm{SD}=429), F_{(1,31)}=4.8, p<0.05$. Isolated and incongruent items did not differ $(F<1)$.
The same ANOVA was run with the data from literate participants. Main effect were found for Congruence, $F_{(2,50)}=11.23$, $p<0.01$, and Material, $F_{(1,25)}=32.66, p<0.01$. Isolated items $(443 \mathrm{~ms}, \mathrm{SD}=186)$ were faster than congruent $(458 \mathrm{~ms}$, $\mathrm{SD}=183)$ and incongruent items $(461 \mathrm{~ms}, \mathrm{SD}=187)$, which did not differ. For Materials, letters $(433 \mathrm{~ms}, \mathrm{SD}=177)$ were responded to faster than shapes $(476 \mathrm{~ms}, \mathrm{SD}=191)$. An interaction was found between Material and Congruence, $F_{(2,50)}=4.89, p<0.01$, due to a negative congruence effect for letters and a positive congruence effect for shapes: Participants were faster with isolated letters $(421 \mathrm{~ms}, \mathrm{SD}=178)$ than with incongruent letters, $F_{(1,25)}=7.99, p<0.01$; Incongruent letters, in turn, were responded to faster than congruent ones $(444 \mathrm{~ms}, \mathrm{SD}=175), F_{(1,25)}=10.79, p<0.01$. Also for shapes, a Congruence effect was observed, $F_{(2,50)}=5.69, p<0.01$. Participants responded faster to isolated $(467 \mathrm{~ms}, \mathrm{SD}=191)$ than to incongruent shapes $(487 \mathrm{~ms}, \mathrm{SD}=195), F_{(2,50)}=8.32$, $p<.001$ but not significantly faster than to congruent ones. Congruent shape $(473 \mathrm{~ms}, \mathrm{SD}=186)$ were responded to faster than incongruent ones, $F_{(2,50)}=6.04, p<0.05$. Results for both groups are displayed in Figure 4.

\section{DISCUSSION}

Amongst visual configurations, letters are special; practice and familiarity make that letters are processed much more efficiently than non-letter configurations of similar complexity (van Leeuwen and Lachmann, 2004; Burgund et al., 2006; Lachmann and van Leeuwen, 2007). In addition, letters have a specific functional relationship to phonemic representations, a relationship

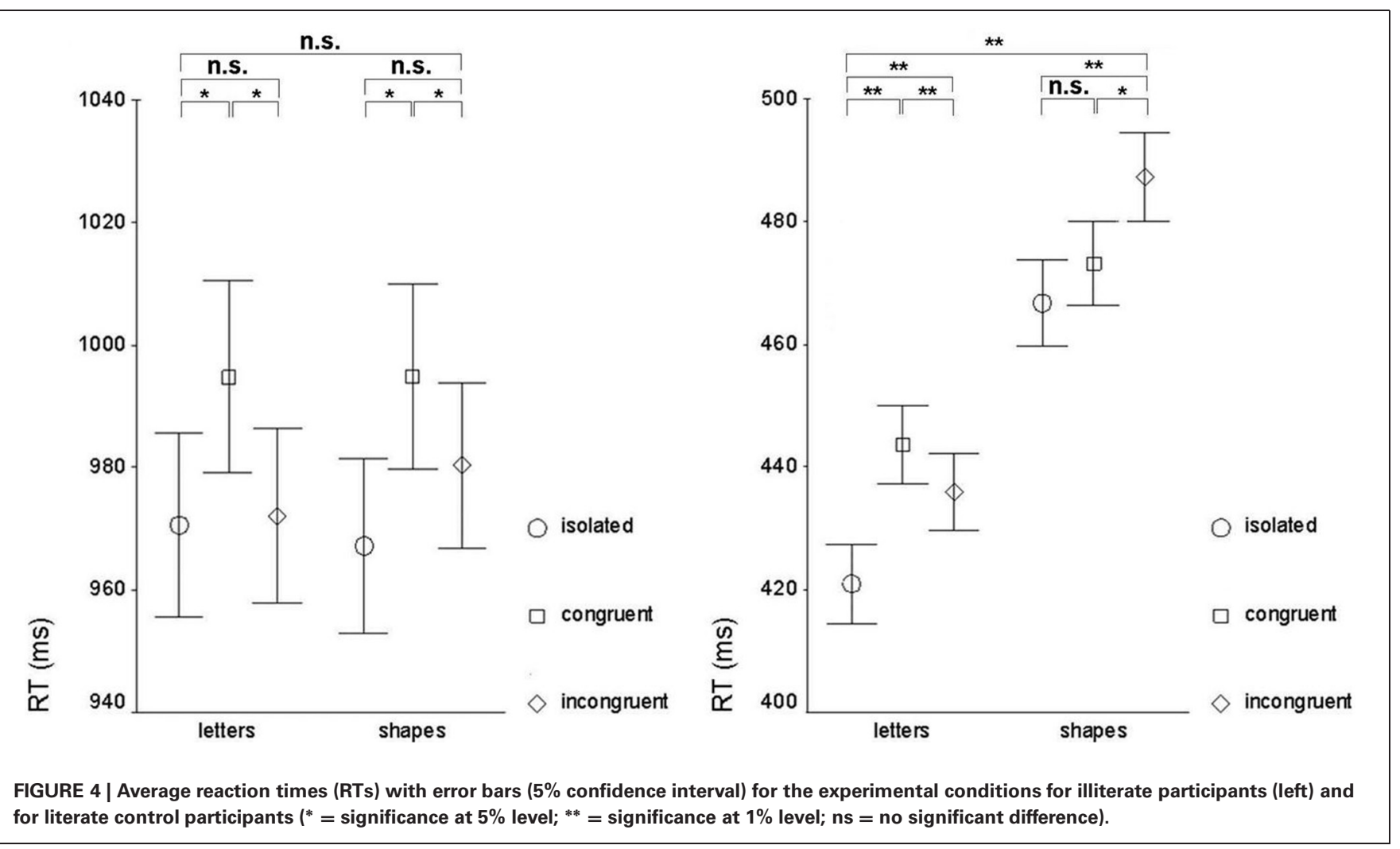


which is developed and automated while children learn to read (Frith, 1985). Recent studies have shown that this development leads to enhanced differentiation and responsiveness of the visual cortices to both orthographic and non-orthographic materials (Dehaene and Cohen, 2007; Dehaene et al., 2010a,b; Cantlon et al., 2011; Pegado et al., 2011). The differentiation gives a new role to established perceptual skills (Dehaene and Cohen, 2007; Lachmann and van Leeuwen, 2007; Blomert, 2011; Perea et al., 2011) which, after modification, need to be coordinated in order to guarantee fast and accurate reading (Lachmann, 2002). To automate this functional coordination, which in the end changes processing of both linguistic and non-linguistic stimuli (Lachmann and van Leeuwen, 2007; Dehaene et al., 2010b; Kolinsky et al., 2011), takes years of reading experience. We addressed the question: what has changed to our visual object perception, once this automatization process is completed?

We performed an experiment with illiterate adults, using the method by which in literates a differentiation between holistic non-letter processing and analytic letter processing was originally shown (van Leeuwen and Lachmann, 2004). This differentiation involves the way the letters are perceived in their immediate surroundings, not necessarily for how strongly their features are bound together at the within-object level (Lachmann and van Leeuwen, 2008b). The latter may depend on the Goodness of the object (Wagemans, 1993, 1999; van der Helm and Leeuwenberg, 1996), e.g., the symmetry of the letter "A." On the other hand, observers tend to ignore symmetry in letters (Lachmann and van Leeuwen, 2007), suggesting that letters are also processed less holistically at this level. Neither does the differentiation in holistic processing at any of these levels have implications for the next higher level, which for letters would be that of morphemes or words. Our claim that letters are processed less holistically than non-letters, therefore, is not in conflict with the well-known word-superiority effect (Reicher, 1969). This effect applies at the level of groupings between letters. It could be argued that, in fact, recognition at this level might benefit from non-holistic processing at our current level (Freeman et al., 2003); word-level processes, for instance, will have difficulty matching individual letters of which the features have mistakenly been bound, based on pre-semantic information, to their surroundings. In sum, therefore, our claim of a distinction in holistic processing between letters and non-letters belongs exclusively to the level of visual integration between these objects and their immediate surroundings.

Even though both groups in the present experiment were of the same ethnicity they are likely to differ in more than their ability to read; such as in general intelligence (e.g., Neubauer and Fink, 2011), education and schooling (e.g., Tun and Lachman, 2008; Ventura et al., 2008), language skills (e.g., bilingualism; Martin-Rhee and Bialystok, 2008) and their familiarity with computers, which all may have influenced their performance. In particular, the latter factor may be responsible for the considerably higher reaction times over-all in illiterates compared to literates. Such differences are probably inevitable in these kinds of studies. However, the effects observed in our earlier studies in literates of different ethnicity and background contrast in the same, consistent manner with those in our illiterates. This may suggest that congruence effects are affected by literacy.

Illiterates not only were equally fast over all in processing letters and shapes, they also showed equally for both faster responses to targets presented in isolation versus in surrounding, as well as same congruence effects of the surroundings across letters and non-letters. For both letters and non-letters, incongruent surroundings led to faster responses than congruent ones.

In contrast, literates of the same ethnicity differentiated between letters and non-letters, just as groups of skilled readers of other ethnicities did (Lachmann and van Leeuwen, 2004; Jincho et al., 2008). They also showed a letter superiority effect that, not surprisingly, is absent in illiterates: letters are processed faster than non-letters and produce opposite congruence effects: positive congruence effects for non-letters, negative congruence effects for letters.

The negative congruence effect in illiterates, i.e., their preference for incongruent surroundings, implies that an analytic perceptual strategy prevailed. Developmental studies might have led us to expect that before reading is automated, at least in an age up from six years on, a holistic strategy to predominate (Schwarzer, 2002). Lachmann and van Leeuwen (2008a) compared adults with different groups of children: beginning normal readers from Grade 3 and 4, and age matched developmental dyslexics. Most of these children showed positive congruence effects for both letters and non-letters, indicating holistic preference. One reason could be that certain brain functions related to reading, especially auditory processing (Banai and Ahissar, 2006) are still developing at this age (Courchesne, 1978; Cheour et al., 2000; Shafer et al., 2000; Ceponiene et al., 2001; Parviainen et al., 2006, 2011; Bruder et al., 2010; Wetzel et al., 2011). This may keep them from using an analytic letter processing strategy that would enable rapid grapheme to phoneme encoding.

A subgroup of dyslexic children in Lachmann and van Leeuwen (2008a; see also Lachmann et al., 2010), with particular difficulties in reading non-words, however, show particularly strong negative congruence effects for letters. This suggests that at this stage in development the analytic strategy is at least present, even though, for object recognition and face recognition, the holistic strategy became already dominant (Schwarzer, 2002).

The results in dyslexics, therefore, are not inconsistent with those of illiterates: the negative congruence effects in illiterates means that analytic processing is not a reading-specific, secondary differentiation in perceptual organization that accompanies the process of learning to read. Rather, it is a generic and primary perceptual processing strategy, on a par with the holistic strategy (Schwarzer et al., 2010). Skilled reading recruits this general perceptual strategy for letter recognition, and uses it in a coordinated fashion along with other functions, including phonological, cognitive, motor, and attentional ones, in meeting the specific demands of reading. What is specific to skilled reading is not the automatization of a letter-specific perceptual strategy (Grainger et al., 2010; Pegado et al., 2011), but the (automated) coordination of various functional components specific to reading.

In this process, letter processing becomes habitually tied up with the analytic perceptual processing strategy. As a result, adult readers tend to no longer process simple non-letter objects 
analytically. This result is in accordance with the pervasiveness of congruency effects in visual object perception (Eriksen and Schultz, 1979; Pomerantz et al., 1989; Boenke et al., 2009). In incongruent conditions, observers fail to ignore irrelevant information, even if this would facilitate processing. This effect is usually considered a result of attentional interference of the irrelevant flanking or surrounding information; this remains a puzzle if we consider that, in principle, focused attention could have been applied to the target (Miles and Proctor, 2010). The present study suggests that this is because analytic processing has become associated with reading. Despite this, having learned to read does not render impossible the analytic processing of non-letter shapes. Evidence of analytic processing is not restricted to letters; negative congruence effects, although sparse, are found whenever active suppression of surrounding information is needed to distinguish a target (Briand, 1994; van Leeuwen and Bakker, 1995; Bavelier et al., 2000). Conversely, there is also evidence of holistic

\section{REFERENCES}

Banai, K., and Ahissar, M. (2006). Auditory processing deficits in dyslexia: task or stimulus related? Cereb. Cortex 16, 1718-1728.

Bavelier, D., Deruelle, C., and Proksch, J. (2000). Positive and negative compatibility effects. Percept. Psychophys. 62, 100-112.

Blomert, L. (2011). The neural signature of orthographic-phonological binding in successful and failing reading development. Neuroimage 57, 695-703.

Boenke, L., Ohl, F., Nikolaev, A., Lachmann, T., and van Leeuwen, C. (2009). Different time courses of Stroop and Garner effects in perception - an event-related potentials study. Neuroimage 45, 1272-1288.

Briand, K. A. (1994). Selective attention to global or local-structure of objects - alternative measures of nontarget processing. Percept. Psychophys. 55, 562-575.

Bruder, J., Leppanen, P. H. T., Bartling, J., Csepe, V., Demonet, J. F., and Schulte-Koerne, G. (2010). An investigation of prototypical and atypical within-category vowels and non-speech analogues on cortical auditory evoked related potentials (AERPs) in 9 year old children. Int. J. Psychophysiol. 79, 106-117.

Burgund, E. D., Guo, Y., and Aurbach, E. L. (2009). Priming for letters and pseudoletters in mid-fusiform cortex: examining letter selectivity and case invariance. Exp. Brain Res. 193, 591-601.

Burgund, E. D., Schlaggar, B. L., and Petersen, S. E. (2006). Development of letter-specific processing: the effect of reading ability. Acta Psychol. $122,99-108$.
Cantlon, J. F., Pinel, P., Dehaene, S., and Pelphrey, K. A. (2011). Cortical representations of symbols, objects, and faces are pruned back during early childhood. Cereb. Cortex 21, 191-199.

Ceponiene, R., Shestakova, A., Balian, P., Alku, P., Yiaguchi, K., and Näätänen, R. (2001). Childrens auditory event-related potentials index sound complexity and speechness. Int. J. Neurosci. 109, 245-260.

Cheour, M., Leppänen, P. H. T., and Kraus, N. (2000). Mismatch negativity (MMN) as a tool for investigating auditory discrimination and sensory memory in infants and children. Clin. Neurophysiol. 111, 4-16.

Courchesne, E. (1978). Neurophysiological correlates of cognitive development: changes in long-latency event-related potentials from childhood to adulthood. Electroencephalogr. Clin. Neurophysiol. 45, 468-482.

Dehaene, S., and Cohen, L. (2007). Cultural recycling of cortical maps. Neuron 56, 384-398.

Dehaene, S., Nakamura, K., Jobert, A., Kuroki, C., Ogawa, S., and Cohen, L. (2010a). Why do children make mirror errors in reading? Neural correlates of mirror invariance in the visual word form area. Neuroimage 49, 1837-1848.

Dehaene, S., Pegado, F., Braga, L. W., Ventura, P., Nunes, G., Jobert, A., Dehaene Lambertz, G., Kolinsky, R., Morais, J., and Cohen, L. (2010b). How learning to read changes the cortical networks for vision and language. Science 330, 1359-1364.

Eriksen, C. W., and Schultz, D. W. (1979). Information processing

processing of letters if the task requires this (van Leeuwen and Lachmann, 2004). This implies that the association of analytic processing and letters is highly context-specific. The data suggest a gradual tendency for a specific, learned processing strategy to predominate for letters.

\section{ACKNOWLEDGMENTS}

Many thanks are due to Andrea Prölß and Andreas Schmitt (University of Kaiserslautern) for their assistance in the analyses of the paper-and-pencil-test and proof reading, to Rosana Tristão and Paul Nealen for helpful comments on earlier drafts of the manuscript, and to all colleagues and officials in India who helped us perform this study. Cees van Leeuwen was supported by an Odysseus grant from the Flemish Science Organization, FWO. Gunjan Khera was supported by a grant from the graduated studies program of the State of Rhineland-Palatinate (Germany).

in visual search: a continuous flow conception and experimental results. Percept. Psychophys. 25, 249-263.

Freeman, E. D., Driver, J., Sagi, D., and Zhaoping, L. (2003). Top-down modulation of lateral interactions in early vision: does attention affect integration of the whole or just perception of the parts? Curr. Biol. 11, 985-989.

Frith, U. (1985). "Beneath the surface of developmental dyslexia," in Surface Dyslexia; Neuropsychological and Cognitive Studies of Phonological Reading, eds K. Patterson, J. Marshall, and M. Coltheart (London, UK: Erlbaum), 301-330.

Grainger, J., Tydgat, I., and Issele, J. (2010). Crowding affects letters and symbols differently. J. Exp. Psychol. Hum. Percept. Perform. 36, 673-688.

Jincho, N., Lachmann, T., and van Leeuwen, C. (2008). Dissociating congruence effects in letters versus shapes: Kanji and kana. Acto Psychol. 129, 138-146.

Kolinsky, R., Verhaeghe, A., Fernandes, T., Mengarda, E. J., Grimm-Cabral, L., and Morais, J. (2011). J. Exp. Psychol. Gen. 140, 210-238.

Lachmann, T. (2002). "Reading disability as a deficit in functional coordination and information integration," in Basic Functions of Language, Reading and Reading Disability, eds E. Witruk, A. D. Friederici, and T. Lachmann (Boston, MA: Kluwer), 165-198.

Lachmann, T., and van Leeuwen, C. (2004). Negative congruence effects in letter and pseudo-letter recognition: the role of similarity and response conflict. Cogn. Process. 5, 239-248.
Lachmann, T., and van Leeuwen, C. (2007). Paradoxical enhancement of letter recognition in developmental dyslexia. Dev. Neuropsychol. 31, 61-77.

Lachmann, T., and van Leeuwen, C. (2008a). Different letter-processing strategies in diagnostic subgroups of developmental dyslexia. Cogn. Neuropsychol. 25, 730-744.

Lachmann, T., and van Leeuwen, C. (2008b). Differentiation of holistic processing in the time course of letter recognition. Acta Psychol. 129, 121-129.

Lachmann, T., Schumacher, B., and van Leeuwen, C. (2009). Controlled but independent: effects of mental rotation and developmental dyslexia in dual task settings. Perception 38 1019-1034.

Lachmann, T., Steinbrink, C., Schumacher, B., and van Leeuwen, C. (2010). Different letterprocessing strategies in diagnostic subgroups of developmental dyslexia occur also in a transparent orthography: reply to a commentary by Spinelli et al. Cogn . Neuropsychol. 26, 759-768.

Martin-Rhee, M. M., and Bialystok, E. (2008). The development of two types of inhibitory control in monolingual and bilingual children. Biling.-Lang. Cogn. 11, 81-93.

Miles, J. D., and Proctor, R. W. (2010). Attention is required for acquisition but not expression of new response biases. J. Exp. Psychol. Learn. Mem. Cogn. 36, 1554-1560.

Neubauer, A., and Fink, A. (2011). Intelligence and neural efficiency: measures of brain activation versus measures of functional connectivity in the brain. Intelligence 37 , 223-229. 
Parviainen, T., Helenius, P., Poskiparta, E., Niemi, P., and Salmelin, R. (2006). Cortical sequence of word perception in beginning readers. J. Neurosci. 26, 6052-6061.

Parviainen, T., Helenius, P., Poskiparta, E., Niemi, P., and Salmelin, R. (2011). Speech perception in the child brain: cortical timing and its relevance to literacy acquisition. Hum. Brain Mapp. 32, 2193-2206.

Pegado, F., Nakamura, K., Cohen, L., and Dehaene, S. (2011). Breaking the symmetry: mirror discrimination for single letters but not for pictures in the Visual Word Form Area. Neuroimage 55, 742-774.

Perea, M., Moret-Tatay, C., and Panadero, V. (2011). Suppression of mirror generalization for reversible letters: evidence from masked priming. J. Mem. Lang. 65, 237-246.

Pomerantz, J. R., and Pristach, E. A. (1989). Emergent features, attention, and perceptual glue in visual form perception. J. Exp. Psychol. Hum. Percept. Perform. 15, 635-649.

Pomerantz, J. R., Pristach, E. A., and Carson, C. E. (1989). "Attention and object perception" in Object Perception: Structure and Process, eds B. Shepp and S. Ballesteros (Hillsdale, NJ: Erlbaum), 53-89.

Reicher, G. M. (1969). Perceptual recognition as a function of meaningfulness of stimulus material. J. Exp. Psychol. 81, 275-280.

Schwarzer, G. (2002). Processing of facial and non-facial visual stimuli in 2-5-year-old children. Infant Child Dev. 11, 253-269.

Schwarzer, G., Kretzer, M., Wimmer, D., and Jovanovic, B. (2010). Holistic face processing among school children, younger and older adults. Eur. J. Dev. Psychol. 7, 511-528.

Serniclaes, W., Ventura, P., Morais, J., and Kolinsky, R. (2005). Categorical perception of speech sounds in illiterate adults. Cognition 98, 35-44.

Shafer, V. L., Morr, M. L., Kreuzer, J. A., and Kurtzberg, D. (2000). Maturation of mismatch negativity in school-age children. Ear Hear. 21, 242-251.

Tun, P. A., and Lachman, M. E. (2008). Age differences in reaction time and attention in a national telephone sample of adults: education, sex, and task complexity matter. Dev. Psychol. 44, 1421-1429. van der Helm, P. A., and Leeuwenberg, E. L. J. (1996). Goodness of visual regularities: a nontransformational approach. Psychol. Rev. 103, 429-456.

van Leeuwen, C., and Bakker, L. (1995). Stroop can occur without Garner interference: strategic and mandatory influences in multidimensional stimuli. Percept. Psychophys. 57, 379-392.

van Leeuwen, C., and Lachmann, T. (2004). Negative and positive congruence effects in letters and shapes. Percept. Psychophys. 6 , 908-925.

Ventura, P., Pattamadilok, C. Fernandes, T., Klein, O., Morais, J., and Kolinsky, R. (2008). Schooling in western culture promotes context-free processing. J. Exp. Child Psychol. 100, 79-88.

Wagemans, J. (1993). Skewed symmetry: a nonaccidental property used to perceive visual forms. J. Exp. Psychol. Hum. Percept. Perform. 19, 364-380.

Wagemans, J. (1999). Toward a better approach to goodness: comments on van der Helm and Leeuwenberg (1996). Psychol. Rev. 106, 610-621.
Wetzel, N., Widmann, A., and Schröger, E. (2011). Processing of novel identifiablility and duration in children and adults. Biol. Sci. 86, 39-49.

Conflict of Interest Statement: The authors declare that the research was conducted in the absence of any commercial or financial relationships that could be construed as a potential conflict of interest.

Received: 02 December 2011; accepted: 25 May 2012; published online: 12 June 2012.

Citation: Lachmann $T$, Khera G Srinivasan N and van Leeuwen C (2012) Learning to read aligns visual analytical skills with grapheme-phoneme mapping: evidence from illiterates. Front. Evol. Neurosci. 4:8. doi: 10.3389/fnevo 2012.00008

Copyright (c) 2012 Lachmann, Khera, Srinivasan and van Leeuwen. This is an open-access article distributed under the terms of the Creative Commons Attribution Non Commercial License, which permits non-commercial use, distribution, and reproduction in other forums, provided the original authors and source are credited. 